\title{
A NOTE ON THE AUTOMORPHISM GROUPS OF LIE MODULE TRIPLE SYSTEMS
}

\author{
NORA C. HOPKINS
}

\begin{abstract}
Using representation theory and well-known facts about automorphism groups of reductive Lie algebras, the automorphism group of a basic Lie module triple system $(M,\{,\},, \mathcal{L}, b, \phi)$ over an algebraically closed field of characteristic zero is related to the automorphism group of the Lie algebra $\mathcal{L}$, the automorphism group of the standard embedding $S$ of $(M,\{,\}$,$) , and$ the automorphism group of the split null extension $\bar{S}$ of $\mathcal{L}$ by $M$.
\end{abstract}

1. Introduction. The problem considered in this paper is that of determining the automorphism group $\operatorname{Aut}(M,\{,\}$,$) of a basic Lie module triple system$ (LMTS) $(M,\{,\},, \mathcal{L}, b, \phi)$ over an algebraically closed field $k$ of characteristic zero. We will do this by relating $\operatorname{Aut}(M,\{,\}$,$) to the automorphism group Aut \mathcal{L}$ of the reductive Lie algebra $\mathcal{L}$ and using well-known facts about automorphism groups of reductive Lie algebras (see $\S 2$ for definitions).

The relationship between $\operatorname{Aut}(M,\{,\}$,$) and Aut \mathcal{L}$ is seen by defining two algebra structures $S$, the standard embedding of $(M,\{,\}$,$) , and \bar{S}$, the split null extension of $\mathcal{L}$ by $M$, on $M \oplus \mathcal{L}$. The groups $\operatorname{Aut}(S, \sigma)$ and $\operatorname{Aut}(\bar{S}, \sigma)$ of automorphisms of $S$ and $\bar{S}$, respectively, stabilizing $M$ and $\mathcal{L}$ have natural restriction maps $r: \operatorname{Aut}(S, \sigma) \rightarrow \operatorname{Aut} \mathcal{L}$ and $\bar{r}: \operatorname{Aut}(\bar{S}, \sigma) \rightarrow$ Aut $\mathcal{L}$ which are homomorphisms. Since $S$ is generated by $(M,\{,\}$,$) it is not surprising that \operatorname{Aut}(M,\{,\},) \cong \operatorname{Aut}(S, \sigma)$ and so $\operatorname{Aut}(M,\{\}$,$) will be determined once the kernel and image of r$ are known. However, since $\bar{S}$ only involves the $\mathcal{L}$-module structure of $M$, the kernel and image of $\bar{r}$ are much easier to compute using standard representation theory facts (Theorems 3.1 and 3.2 and Lemma 4.1). Since $\operatorname{Aut}(S, \sigma) \subseteq \operatorname{Aut}(\bar{S}, \sigma)$, using Lemma 3.5, Corollary 3.6, and Lemma 4.2 it is possible to determine the kernel and image of $r$ from those of $\bar{r}$ (Theorems 3.7 and 3.9 and Lemma 4.3). The surprising conclusion is that if $\phi$ is symmetric or symplectic, the images of $r$ and $\bar{r}$ are the same (Corollary 3.8), $\operatorname{Aut}(S, \sigma)$ is a normal subgroup of $\operatorname{Aut}(\bar{S}, \sigma)$, and $\operatorname{Aut}(\bar{S}, \sigma) / \operatorname{Aut}(S, \sigma) \cong k^{*}$ (Theorem 4.4).

This paper contains material from my dissertation, done at the University of Virginia under the direction of J. R. Faulkner, to whom I owe great thanks for his help. I also thank J. C. Ferrar for his comments on the material herein.

2. Basic definitions. Recall from [3] that a Lie module triple system (abbreviated LMTS) is formed from a finite-dimensional Lie algebra $\mathcal{L}$ having a nondegenerate symmetric associative bilinear form $b$ and a finite-dimensional faithful $\mathcal{L}$-module $M$ having a nondegenerate $\mathcal{L}$-invariant bilinear form $\phi$, that is

$$
\phi(x l, y)=-\phi(x, y l)
$$

Received by the editors October 10, 1985.

1980 Mathematics Subject Classification (1985 Revision). Primary 17A40, 20F20; Secondary 17B60, $17 \mathrm{~B} 40$. 
for all $x, y \in M, l \in \mathcal{L}$. A triple product $\{,$,$\} on M$ is defined by setting $\{x y z\}:=x R(y, z)$ where $R: M \times M \rightarrow \mathcal{L}$ is given by

$$
b(l, R(y, z))=\phi(z l, y)
$$

for all $y, z \in M, l \in \mathcal{L}$. The Lie module triple system $(M,\{,\}$,$) is denoted$ $(M,\{,\},, \mathcal{L}, b, \phi)$ when the ingredients need to be specified.

We will always assume that $(M,\{,\},, \mathcal{L}, b, \phi)$ is a simple LMTS such that $M$ is completely reducible as an $\mathcal{L}$-module. Hence $\mathcal{L}$ is reductive (Theorem 10, p. 81 of $[4])$, i.e. $\mathcal{L}=[\mathcal{L}, \mathcal{L}] \oplus C$ with $[\mathcal{L}, \mathcal{L}]$ semisimple and $C$ a central ideal of $\mathcal{L}$ whose elements act semisimply on $M$. $\Lambda$ will denote the set of lowest weights, without multliplicities, of $M$ as an $\mathcal{L}$-module. We will usually assume that $(M,\{,\},, \mathcal{L}, b, \phi)$ is one of the following basic types of LMTS's:

Type (I). $M$ is an irreducible self-dual $\mathcal{L}$-module of lowest weight $\lambda, \mathcal{L}$ is semisimple, and $\phi$ is symmetric or symplectic.

Type (II). $M=M_{1} \oplus M_{2}$ with $M_{1}$ and $M_{2}$ isomorphic irreducible self-dual $\mathcal{L}$ modules of lowest weight $\lambda, \mathcal{L}$ is semisimple, $\phi$ is symmetric or symplectic, and $\phi$ restricted to $M_{i} \times M_{i}$ is identically zero for $i=1,2$. $\operatorname{Der}(M,\{,\})=,\mathcal{L} \oplus \operatorname{sl}(2)$ where $\operatorname{Der}(M,\{,\}$,$) is the derivation algebra of (M,\{,\}$,$) and \mathcal{L}, \operatorname{sl}(2)$ are ideals of $\operatorname{Der}(M,\{,\}$,$) .$

Type (III). $M=M_{1} \oplus M_{2}$ with $M_{1}$ and $M_{2}$ irreducible dual $\mathcal{L}$-submodules of lowest weight $\lambda_{1}$ and $\lambda_{2}$ respectively with $\lambda_{1} \neq \lambda_{2}$. $\phi$ restricted to $M_{i} \times M_{i}$ is identically zero and there is a $z \in k^{*}$ with $\phi(z, y)=a \phi(y, z)$ for all $z \in M_{1}$, $y \in M_{2}$. $\mathcal{L}$ is semisimple or has a one-dimensional center spanned by $c$ where $\left(x_{1}+x_{2}\right) c:=x_{1}-x_{2}$ for $x_{i} \in M_{i}$.

Note that simple Lie triple systems are basic $[\mathbf{5}, \mathbf{1}]$. Other examples of basic LMTS's are given in $[\mathbf{1}, \mathbf{2}]$ where it was shown that if $(M,\{,\},, \mathcal{L}, b, \phi)$ is a LMTS for which $M$ is a completely reducible $\mathcal{L}$-module and $\phi$ is symmetric or symplectic, then $(M,\{,\}$,$) can be constructed from basic LMTS's and one- and$ two-dimensional abelian LMTS's. Note however that not all simple completely reducible LMTS's are basic (see [1] for examples of nonbasic simple LMTS's).

$\alpha \in \mathrm{GL}(M)$ is an automorphism of $(M,\{,\}$,$) if \{x y z\} \alpha=\{x \alpha, y \alpha, z \alpha\}$ and $\operatorname{Aut}(M,\{,\}$,$) will denote the automorphism group of (M,\{,\},, \mathcal{L}, b, \phi)$. There are two nonassociative algebra structures naturally defined on $M \oplus \mathcal{L}$, the first being the split null extension $\bar{S}$ of $\mathcal{L}$ by $M$, with multiplication defined by $(x+l)\left(y+l_{1}\right):=$ $x l_{1}-y l+\left[l, l_{1}\right]$, and the second being the standard embedding $S$ of $(M,\{,\}$, (see [3]), with multiplication defined by $(x+l)\left(y+l_{1}\right):=x l_{1}-y l+\left[l, l_{1}\right]+R(x, y)$ for $x, y \in M, l, l_{1} \in S$. $\sigma \in \operatorname{GL}(M \oplus \mathcal{L})$ defined by $(x+l) \sigma:=-x+l$ is an automorphism of both $S$ and $\bar{S}$ fixing $\mathcal{L}$. Let $\operatorname{Aut}(S, \sigma)($ respectively $\operatorname{Aut}(\bar{S}, \sigma)$ ) be the automorphisms of $S$ (respectively $\bar{S}$ ) which commute with $\sigma$.

Clearly $\operatorname{Aut}(S, \sigma)$ is a subgroup ofAut $(\bar{S}, \sigma)$ and $\psi: \operatorname{Aut}(M,\{,\},) \rightarrow \operatorname{Aut}(S, \sigma)$ defined by $(x+l)(\tau \psi):=x \tau+\left(\tau^{-1} l \tau\right)$ for $\tau \in \operatorname{Aut}(M,\{,\}$,$) is an isomor-$ phism. For computing $\operatorname{Aut}(\bar{S}, \sigma) / \operatorname{Aut}(S, \sigma)$ we will also need to consider the automorphism group Aut $\mathcal{L}$ of $\mathcal{L}$ and the homomorphisms $r: \operatorname{Aut}(\mathcal{S}, \sigma) \rightarrow$ Aut $\mathcal{L}$ and $\bar{r}: \operatorname{Aut}(\bar{S}, \sigma) \rightarrow$ Aut $\mathcal{L}$ given by restriction, i.e. $\tau r:=\left.\tau\right|_{\mathcal{L}}$ and $\eta \bar{r}:=\left.\eta\right|_{\mathcal{L}}$ for $\tau \in \operatorname{Aut}(S, \sigma), \eta \in \operatorname{Aut}(\bar{S}, \sigma)$. The kernel of $r$ (respectively $\bar{r}$ ) is denoted ker $r$ (respectively $\operatorname{ker} \bar{r}$ ) and the image of $r$ (respectively $\bar{r}$ ) by im $r($ respectively $\operatorname{im} \bar{r}$ ). 
3. Images. Since any automorphism of $\mathcal{L}$ is the product of an inner automorphism and one that stabilizes a Borel subalgebra of $\mathcal{L}[\mathbf{4}]$, we will consider these two types of automorphisms of $\mathcal{L}$ separately. Inn Aut $\mathcal{L}$ will be the group of inner automorphisms of $\mathcal{L}$. For $e \in \mathcal{L}$ nilpotent define $\exp \left(\operatorname{ad}_{S} e\right) \in \operatorname{Aut}(S, \sigma)$ by

$$
\exp \left(\operatorname{ad}_{s} e\right):=\sum_{n=0}^{\infty} \frac{1}{n !}\left(\operatorname{ad}_{s} e\right)^{n} \quad \text { where } x\left(\operatorname{ad}_{s} e\right):=x e \text { for all } x \in S \text {. }
$$

TheOREM 3.1 (COROLlaRY 5, p. 44 OF [6]). Let Inn $\operatorname{Aut}(S, \sigma)$ be the subgroup of $\operatorname{Aut}(S, \sigma)$ generated by $\left\{\exp \left(a d_{S} e\right) \mid e \in \mathcal{L}\right.$, e nilpotent $\}$. Then $\operatorname{tr} \in$ Inn Aut $\mathcal{L}$ for $\tau \in \operatorname{Inn} \operatorname{Aut}(S, \sigma), r: \operatorname{Inn} \operatorname{Aut}(S, \sigma) \rightarrow \operatorname{Inn}$ Aut $\mathcal{L}$ is onto, and its kernel is isomorphic to $\Lambda_{w} / \Lambda_{r}$ where $\Lambda_{r}$ is the root lattice of $[\mathcal{L}, \mathcal{L}]$ and $\Lambda_{w}$ is the weight lattice of $M$ as an $[\mathcal{L}, \mathcal{L}]$-module. Thus $\operatorname{Inn}$ Aut $\mathcal{L} \subseteq \operatorname{im} r \subseteq \operatorname{im} \bar{r}$.

Thus if $\nu \in \operatorname{Aut}(\bar{S}, \sigma), \nu=\eta_{1} \eta_{2}$ where $\eta_{1} \in \operatorname{Inn} \operatorname{Aut}(S, \sigma)$ and $\eta_{2} \bar{r}$ stabilizes a Borel subalgebra of $\mathcal{L}$.

Now suppose $\tau \in$ Aut $\mathcal{L}$ stabilizes a Borel subalgebra $B$ of $\mathcal{L}$. Then it stabilizes the Cartan subalgebra $H$ contained in $B$, and if $\tau^{*}: H^{*} \rightarrow H^{*}$ is defined by $h\left(\mu \tau^{*}\right):=\mu\left(h \tau^{-1}\right)$ for $h \in H, \mu \in H^{*}$, then $\alpha \tau^{*}$ is a positive root if $\alpha$ is a positive root and $\Delta \tau^{*}=\Delta$ for $\Delta$ a base of the positive roots where $\Delta \tau^{*}:=\left\{\alpha \tau^{*} \mid \alpha \in \Delta\right\}$.

THEOREM 3.2. Suppose $\tau \in$ Aut $\mathcal{L}$ stabilizes a Borel subalgebra $B$ of $\mathcal{L}$ and $N_{i}$ is the $\lambda_{i}$ weight space relative to $B$ for $\lambda_{i} \in \Lambda=\left\{\lambda_{1}, \ldots, \lambda_{l}\right\}$. Then $\tau \in \operatorname{im} \bar{r}$ if and only if $\Lambda \tau^{*}=\Lambda$ and $\operatorname{dim} N_{i}=\operatorname{dim} N_{j}$ if $\lambda_{i} \tau^{*}=\lambda_{j}$.

Proof. Suppose $\eta \in \operatorname{Aut}(\bar{S}, \sigma)$ with $\eta \bar{r}=\tau$. Then if $\mu$ is a weight of $M$ as an $\mathcal{L}$-module and $M_{\mu}$ is the $\mu$ weight space of $M, x \in M_{\mu \tau^{*}}$ for $x \in M_{\mu}$. Since $\tau$ permutes positive root spaces, $x \eta$ is a lowest weight vector if and only if $x$ is a lowest weight vector. Thus $\Lambda \tau^{*}=\Lambda$ and $\operatorname{dim} N_{i}=\operatorname{dim} N_{j}$ if $\lambda_{i} \tau^{*}=\lambda_{j}$.

Conversely, suppose $\tau \in$ Aut $\mathcal{L}$ stabilizes $B, \Lambda \tau^{*}=\Lambda$ and $\operatorname{dim} N_{i}=\operatorname{dim} N_{j}$ if $\lambda_{i} \tau^{*}=\lambda_{j}$. Define a new action of $\mathcal{L}$ on $M$ by $x \cdot l:=x(l \tau)$ and denote $M$ with this new $\mathcal{L}$-action by $\tilde{M}$. Now if $x \in M_{\mu}$, then $x \in \tilde{M}_{\mu \tau^{-1}{ }^{*}}$ so $M$ and $\tilde{M}$ have the same lowest weights. Since the $\lambda_{i}$ weight spaces of $M$ and $\tilde{M}$ are the same dimension for $\lambda_{i} \in \Lambda, M$ and $\tilde{M}$ are isomorphic $\mathcal{L}$-modules, i.e. there is a linear bijection $\nu: M \rightarrow \tilde{M}$ with $(x l) \nu=(x \nu) \cdot l=(x \nu)(l \tau)$. Thus $\eta \in \operatorname{GL}(M \oplus \mathcal{L})$ defined by $(x+l) \eta:=x \nu+l \tau$ is in $\operatorname{Aut}(\bar{S}, \sigma)$ and $\eta \bar{r}=\tau$. Hence $\tau \in \operatorname{im}(\bar{r})$.

Note 3.3. If $\eta \in \operatorname{Aut}(\bar{S}, \sigma)$ with $\eta(\bar{r})=\tau$ stabilizing a Borel subalgebra $B$ of $\mathcal{L}$, then $y \eta$ is a highest weight vector of $M$ if and only if $y$ is a highest weight vector. In particular, if $x_{1}$ and $x_{2}$ are nonzero lowest weight vectors with $x_{1} \eta=x_{2}$, let $y_{1}=x_{1} \cdot e_{1} \cdots e_{n}$ be a nonzero highest weight vector and let $y_{2}$ be any nonzero highest weight vector of the irreducible submodule generated by $x_{2}$. Then $y_{1} \eta=\left(x_{1} \eta\right)\left(e_{1} \tau\right) \cdots\left(e_{n} \tau\right)=c y_{2}$ for some $c \in k$ and $c \neq 0$ since $\eta$ is injective.

It is also important to note that if $\left\{x_{i, 1}, \ldots, x_{i, k}\right\}$ is a basis of $N_{i}$ and $\left\{x_{j, 1}^{\prime}, \ldots\right.$, $\left.x_{j, k}^{\prime}\right\}$ is a basis of $N_{j}$, then the $\eta$ constructed in the second half of the proof above can be defined so that $x_{i, n} \eta=x_{j, n}^{\prime}$ for $n=1, \ldots, k$ and $i=1, \ldots, l$. For if $M_{i, n}$ is the irreducible submodule of $M$ generated by $x_{i, n}$ and $\tilde{M}_{j, n}$ is the irreducible submodule of $\tilde{M}$ generated by $x_{j, n}^{\prime}$, then $M_{i, n}$ and $\tilde{M}_{j, n}$ are isomorphic $\mathcal{L}$-modules since $\lambda_{i} \tau^{*}=\lambda_{j}$. Let $\nu_{i, n}: M_{i, n} \rightarrow \tilde{M}_{j, n}$ be such an isomorphism. Adjusting 
$\nu_{i, n}$ by a scalar multiple if necessary, we may assume $x_{i, n} \nu_{i, n}=x_{j, n}^{\prime}$. Letting $M_{i}:=\sum_{n=1}^{k} M_{i, n}$ and $\tilde{M}_{j}=\sum_{n=1}^{k} \tilde{M}_{j, n}$, define $\nu_{i}: M_{i} \rightarrow \tilde{M}_{j}$ by $\left(\sum_{n=1}^{k} z_{n}\right) \nu_{i}:=$ $\sum_{n=1}^{k}\left(z_{n} \nu_{i, n}\right)$ for $z_{n} \in M_{i, n}$ and define $\nu: M \rightarrow \tilde{M}$ by $\left(\sum_{i=1}^{k} z_{i}\right) \nu:=\sum_{i=1}^{l}\left(z_{i} \nu_{i}\right)$. As before $\eta$ is defined by $(x+l) \eta:=x \nu+l \tau$.

Note 3.4. Finally note that if $\eta \in \operatorname{Aut}(\bar{S}, \sigma)$, then $\eta$ is completely determined by $\eta \bar{r}=\tau \in$ Aut $\mathcal{L}$ and the action of $\eta$ on a set of vectors $\left\{x_{1}, \ldots, x_{j}\right\}$ spanning the lowest weight spaces of $M$ since vectors of the form $x_{i} l_{1} \cdots l_{n}$ span $M$ and $\left(x_{i} l_{1} \cdots l_{n}\right)=\left(x_{i} \eta\right)(l \tau) \cdots\left(l_{n} \tau\right)$.

Having determined im $\bar{r}$, the following lemma will allow us to determine im $r$ since $\operatorname{im} r \subseteq \operatorname{im} \bar{r}$. Note that the lemma is true for any LMTS.

LEMMA 3.5. Suppose $\eta \in \operatorname{Aut}(\bar{S}, \sigma)$. Then $\eta \in \operatorname{Aut}(S, \sigma)$ if and only if for all $l \in \mathcal{L}, y, z \in M$

$$
b(l \eta, R(y, z) \eta)=\phi((z l) \eta, y \eta) .
$$

Proof. Suppose $\eta \in \operatorname{Aut}(S, \sigma)$. Then $R(y, z) \eta=R(y \eta, z \eta)$ so by (2.2) $b(l \eta, R(y, z) \eta)=b(l \eta, R(y \eta, z \eta))=\phi((z \eta)(l \eta), y \eta)=\phi((z l) \eta, y \eta)$ giving (3.5.1). Conversely, suppose $\eta \in \operatorname{Aut}(\bar{S}, \sigma)$ satisfies (3.5.1). Then for $u, v \in M$

$$
\begin{aligned}
b(R(u, v) \eta, R(y, z) \eta) & =\phi([z R(u, v) \eta], y \eta)=\phi((z \eta)[R(u, v) \eta], y \eta) \\
& =b(R(u, v) \eta, R(y \eta, z \eta))
\end{aligned}
$$

by $(2.2)$ so $R(y, z) \eta=R(y \eta, z \eta)$ and $\eta \in \operatorname{Aut}(S, \sigma)$.

COROLlary 3.6. Suppose $(M,\{,\}$,$) is basic and \eta \in \operatorname{Aut}(\bar{S}, \sigma)$. Then $\eta \in \operatorname{Aut}(S, \sigma)$ if and only if

$$
\phi(z \eta, y \eta)=\phi(z, y)
$$

for all $y, z \in M$.

Proof. If $\mathcal{L}$ is semisimple, then $b\left(l \tau, l_{1} \tau\right)=b\left(l, l_{1}\right)$ for all $\tau \in$ Aut $\mathcal{L}, l, l_{1} \in \mathcal{L}$ [4]. Thus $b\left(l \eta, l_{1} \eta\right)=b\left(l, l_{1}\right)$ for all $l, l_{1} \in \mathcal{L}$ if $(M,\{,\}$,$) is Type (I) or (II) or$ if $(M,\{,\}$,$) is Type (III) with \mathcal{L}$ semisimple. If $(M\{,\}$,$) is Type (III) with \mathcal{L}$ having a one-dimensional center, Theorem 3.2 implies that either $M_{1} \eta=M_{2}$ and $M_{2} \eta=M_{1}$ or $M_{1} \eta=M_{1}$ and $M_{2} \eta=M_{2}$. In the first case $c \eta=-c$ and in the second case $c \eta=c$ so $b(c \eta, c \eta)=b(c, c)$ and $b\left(l \eta, l_{1} \eta\right)=b\left(l, l_{1}\right)$ for all $l \in \mathcal{L}$ for $(M,\{,\}$,$) any basic LMTS. Then (3.5.1) becomes \phi(z l \eta, y \eta)=b(l \eta, R(y, z) \eta)=$ $b(l, R(y, z))=\phi(z l, y)$ by (2.2) giving (3.6.1) since $M$ is spanned by vectors of the form $z l$.

Since Inn Aut $\mathcal{L} \subseteq \operatorname{im} r$, we again only need to determine which $\tau \in$ Aut $\mathcal{L}$ stabilizing a Borel subalgebra $B$ are in im $r$.

THEOREM 3.7. Suppose $(M,\{\}$,$) is a basic LMTS and \tau \in \operatorname{im} \bar{r}$ stabilizes a Borel subalgebra $B$ of $\mathcal{L}$.

(i) If $\lambda \tau^{*}=\lambda$ for all $\lambda \in \Lambda$, then $\tau \in \operatorname{im} r$.

(ii) If $(M,\{,\}$,$) is Type (III) with a= \pm 1$ and $\lambda_{1} \tau^{*}=\lambda_{2}$, then $\tau \in \operatorname{im} r$.

Proof. We want to find $\eta \in \operatorname{Aut}(\bar{S}, \sigma)$ with $\eta \bar{r}=\tau$ and such that (3.6.1) is satisfied. By Note 3.4 we can do this simply by specifying the images of the lowest weight vectors. 
Suppose $\lambda \tau^{*}=\lambda$. If $(M,\{,\}$,$) is Type (I) let x$ be a nonzero lowest weight vector and $y:=x e_{1} \cdots e_{n}$ be a nonzero highest weight vector. By Note 3.3, $x\left(e_{1} \tau\right) \cdots\left(e_{\eta} \tau\right)=c y$ for some $c \in k^{*}$. By Notes 3.3 and 3.4 we can define $\eta \in$ Aut $(\bar{S}, \sigma)$ by $l \eta:=l \tau$ and $x \eta:=\sqrt{c}^{-1} x$. Then $y \eta=\sqrt{c} y$ and $\phi(x \eta, y \eta)=\phi(x, y)$. If $w$ is a weight vector of $M$ of weight $\mu \neq-\lambda$, by $(2.1) \phi(x, w)=0=\phi(x \eta, w \eta)$ since $w \eta$ has weight $\mu \tau^{*} \neq-\lambda$. Thus $\phi(x \eta, w \eta)=\phi(x, w)$ for all $w \in M$. By induction on $m$

$$
\begin{aligned}
\phi\left(\left(x l_{1}\right.\right. & \left.\left.\cdots l_{m}\right) \eta, w \eta\right)=\phi\left((x \eta)\left(l_{1} \eta\right) \cdots\left(l_{m} \eta\right), w \eta\right) \\
& =-\phi\left((x \eta)(l \eta) \cdots\left(l_{m-1} \eta\right),(w \eta)\left(l_{m} \eta\right)\right)=-\phi\left(x l_{1} \cdots l_{m-1}\right) \eta,\left(w l_{m}\right) \eta \\
& =-\phi\left(x l_{1} \cdots l_{m-1}, w l_{m}\right)=\phi\left(x l_{1} \cdots l_{m}, w\right)
\end{aligned}
$$

so $\eta$ satisfies (3.6.1) and hence $\eta \in \operatorname{Aut}(S, \sigma)$ so $\tau \in \operatorname{im} r$. If $(M\{,\}$,$) is Type$ (II) or (III) Note 3.3 again shows that if $x_{2}$ is a nonzero lowest weight vector of $M_{2}$ and $y_{2}=x_{2} e_{1} \cdots e_{n}$ is a nonzero highest weight vector, then $x_{2}\left(e_{1} \tau\right) \cdots\left(e_{n} \tau\right)=c y_{2}$ for some $c \in k^{*}$. Let $x_{1}$ be a nonzero lowest weight vector of $M_{1}$ and define $\eta \in$ $\operatorname{Aut}(\bar{S}, \sigma)$ by $l \eta:=l \tau, x_{1} \eta:=x_{1}$ and $x_{2} \eta:=c^{-1} x_{2}$. Thus $y_{2} \eta=y_{2}$ so $\phi\left(x_{1} \eta, y_{2} \eta\right)=$ $\phi\left(x_{1}, y_{2}\right)$ and $\phi\left(y_{2} \eta, x_{1} \eta\right)=\phi\left(y_{2}, x_{1}\right)$. By the same induction argument as before $\phi(z \eta, w \eta)=\phi(z, w)$ and $\phi(w \eta, z \eta)=\phi(w, z)$ for all $z \in M_{1}, w \in M_{2}$ giving (3.6.1).

For (ii) let $x_{i} \in M_{i}$ be nonzero lowest weight vectors for $i=1,2$ and $y_{i} \in M_{i}$ be nonzero highest weight vectors for $i=1,2$ with $y_{2}=x_{2} e_{1} \cdots e_{n}$. Then by Note $3.3, x_{1}\left(e_{1} \tau\right) \cdots\left(e_{n} \tau\right)=c y_{1}$ for some $c \in k^{*}$. Also $\phi\left(x_{1}, y_{2}\right)=d \phi\left(x_{2}, y_{1}\right)$ so if we define $\eta \in \operatorname{Aut}(\bar{S}, \sigma)$ by $l \eta:=l \tau, x_{1} \eta:=x_{2}$, and $x_{2} \eta:=d c^{-1} x_{1}$, then $y_{2} \eta=d y_{1}$ and $\phi\left(x_{1} \eta, y_{2} \eta\right)=d \phi\left(x_{2}, y_{1}\right)=\phi\left(x_{1}, y_{2}\right)$ and again using induction we have $\phi(z \eta, w \eta)=\phi(z, w)$ for all $z \in M_{1}, w \in M_{2}$. Since $\phi$ is symmetric or symplectic we also have $\phi(w \eta, z \eta)=\phi(w, z)$ for $z \in M_{1}, w \in M_{2}$ so $\eta$ satisfies (3.6.1).

Corollary 3.8. Suppose $(M\{,\},, \mathcal{L}, b, \phi)$ is a basic LMTS and $\phi$ is symmetric or symplectic. Then $\operatorname{im} r=\operatorname{im} \bar{r}$. Hence $\operatorname{Aut}(\bar{S}, \sigma)=\operatorname{ker} \bar{r} \operatorname{Aut}(S, \sigma)$.

PROOF. The first statement is an immediate consequence of Theorems 3.1, 3.2 and 3.7. For the second one if $\eta \in \operatorname{Aut}(\bar{S}, \sigma)$, there is an $\eta_{1} \in \operatorname{Aut}(S, \sigma)$ with $\eta \bar{r}=\eta_{1} r=\eta_{1} \bar{r}$ so $\eta \eta_{1}^{-1} \in \operatorname{ker} \bar{r}$.

Thus the difference between $\operatorname{Aut}(\bar{S}, \sigma)$ and $\operatorname{Aut}(S, \sigma)$ lies in $\operatorname{ker} \bar{r}$ if $(M\{,\},, \mathcal{L}$, $b, \phi)$ is basic and $\phi$ is symmetric or symplectic. The following theorem finishes the question of determining $\operatorname{im} r$ for $(M\{,\}$,$) a basic LMTS.$

THEOREM 3.9. Suppose ( $M\{$, , \}) is Type (III) with $a \neq \pm 1$ and $\tau \in \operatorname{im} \bar{r}$ stabilizes a Borel subalgebra of $\mathcal{L}$. Then $\tau \in \operatorname{im} r$ if and only if $\lambda_{i} \tau^{*}=\lambda_{i}$ for $i=1,2$.

PROOF. If $\lambda_{i} \tau^{*}=\lambda_{i}$ for $i=1,2 \tau \in \operatorname{im} r$ by Theorem 3.7(i). If $\lambda_{i} \tau^{*} \neq \lambda_{i}$ for $i=1,2$, then $\lambda_{1} \tau^{*}=\lambda_{2}$ and $\lambda_{2} \tau^{*}=\lambda_{1}$ by Theorem 3.2, so if $\eta \in \operatorname{Aut}(S, \sigma)$ with $\eta r=\tau, M_{1} \eta=M_{2}$ and $M_{2} \eta=M_{1}$. But $\eta$ must satisfy (3.6.1), so for $y \in M_{2}$, $z \in M_{1}, \phi(y, z)=\phi(y \eta, z \eta)=a \phi(z \eta, y \eta)=a \phi(z, y)=a^{2} \phi(y, z)$. Thus $a^{2}=1$, i.e. $a= \pm 1$, a contradiction. Hence no such $\eta$ exists. 
4. Kernels. In this section we will determine $\operatorname{ker} \bar{r}$ and $\operatorname{ker} r$.

LEMMA 4.1. Suppose $\Lambda=\left\{\lambda_{1}, \ldots, \lambda_{l}\right\}, N_{i}$ is the $\lambda_{i}$ weight space of $M$ for $i=1, \ldots, l$, and $P=N_{1} \oplus \cdots \oplus N_{l}$. Then $N_{i}$ is $\operatorname{ker} \bar{r}$ invariant and if $\operatorname{dim} N_{i}=n_{i}$ for $i=1, \ldots, l$, then $\psi: \operatorname{ker} \bar{r} \rightarrow \mathrm{GL}\left(n_{1}, k\right) \times \cdots \times \mathrm{GL}\left(n_{l}, k\right)$ defined by $\eta \psi:=\left.\eta\right|_{p}$ is an isomorphism.

PROOF. This follows from the proof of Theorem 3.2 and Notes 3.3 and 3.4 since if $\eta \in \operatorname{ker} \bar{r}, \eta$ certainly fixes a Borel subalgebra of $\mathcal{L}$.

LemMA 4.2. Suppose $\eta \in \operatorname{ker} \bar{r}$. Then $\eta \in \operatorname{ker} r$ if and only if $\eta$ satisfies (3.6.1).

PROOF. If $\eta \in \operatorname{ker} \bar{r}, l \eta=l$ for all $l \in \mathcal{L}$, so $b\left(l \eta, l_{1} \eta\right)=b\left(l, l_{1}\right)$ for all $l, l_{1}$. The rest of the proof is the same as that of Corollary 3.6 using Lemma 4.1 of [3].

LEMмA 4.3. (i) If $(M\{,\}$,$) is Type (I), ker \bar{r} \cong k^{*}$ and $\operatorname{ker} r \cong C_{2}$, the cyclic group of order 2 .

(ii) If $(M\{,\}$,$) is Type (II), \operatorname{ker} \bar{r} \cong \mathrm{GL}(2, k)$ and $\operatorname{ker} r \cong \mathrm{SL}(2, k)$.

(iii) If $(M\{,\}$,$) is Type (III), \operatorname{ker} \bar{r} \cong k^{*} \times k^{*}$ and $\operatorname{ker} r \cong k^{*}$.

PROOF. The assertions about $\operatorname{ker} \bar{r}$ follow from Lemma 4.1 and those about ker $r$ for Types (I) and (III) follow immediately from Lemma 4.2. For Type (II) recall that there is an ideal $K$ of $\operatorname{Der}(M\{,\}$,$) isomorphic to \operatorname{sl}(2)$ and consisting of endomorphisms of $M$ which commute with $\mathcal{L}$, so $P D \subseteq P$ for $D \in \mathcal{K}$ and $P$ the lowest weight space of $M$. Now if $D \in \mathcal{K}, \phi(z D, y)=-\phi(z, y D)$ by Lemma 2.3 of [2]. Hence if $D \in \mathcal{K}$ is nilpotent, $\exp D \in \operatorname{Aut}(M\{,\}$,$) where \exp D:=\mathrm{id}+D$, so $\eta_{D} \in \operatorname{ker} r$ where $(x+l) \eta_{D}:=x(\exp D)+l$. Note that the group generated by $\left\{\eta_{D} \mid D \in \mathcal{K}\right.$ nilpotent $\}$ is isomorphic to $\mathrm{SL}(2, k) \subseteq \mathrm{GL}(2, k)=\operatorname{ker} \bar{r}$ under restriction to $P$. Now $\mathrm{GL}(2, k)=k^{*} \mathrm{SL}(2)$ where $\eta_{b} \in k^{*}$ for $b \in k^{*}$ is defined by $(x+l) \eta_{b}:=b x+l$. Then Lemma 4.2 gives that $\eta_{b} \in \operatorname{ker} r$ if and only if $b= \pm 1$.

THEOREM 4.4. Suppose $(M\{,\},, \mathcal{L}, b, \phi)$ is a basic LMTS and $\phi$ is symmetric or symplectic. Then $\operatorname{Aut}(S, \sigma)$ is a normal subgroup of $\operatorname{Aut}(\bar{S}, \sigma)$ and

$$
\operatorname{Aut}(\bar{S}, \sigma) / \operatorname{Aut}(S, \sigma) \cong k^{*} \text {. }
$$

Proof. For each type ker $\bar{r}=k^{*} \operatorname{ker} r$ where for Types (I) and (III) $\eta_{b} \in k^{*}$ is defined for $b \in k^{*}$ by $(x+l) \eta_{b}:=b x+l$ and for Type (III) $\eta_{b}$ is defined by $\left(x_{1}+x_{2}+l\right) \eta_{b}=b x_{1}+x_{2}+l$ for $x_{i} \in M_{i}$. Hence by Corollary 3.8, $\operatorname{Aut}(\bar{S}, \sigma)=$ $\operatorname{ker} \bar{r} \operatorname{Aut}(S, \sigma)=k^{*} \operatorname{Aut}(S, \sigma)$. For Types (I) and (II) $\eta_{b}^{-1} \nu \eta_{b}=\nu$ for $\nu \in \operatorname{Aut}(S, \sigma)$ and for Type (III) if $\nu \in \operatorname{Aut}(S, \sigma)$ with $M_{i} \nu=M_{i}, i=1,2$, then $\eta_{b}^{-1} \nu \eta_{b}=\nu$ and if $\nu \in \operatorname{Aut}(S, \sigma)$ with $M_{1} \nu=M_{2}, M_{2} \nu=M_{1}$, then $\eta_{b}^{-1} \nu \eta_{b}=\nu \xi$ where $\left(x_{1}+x_{2}+l\right) \xi:=b x_{1}+b^{-1} x_{2}+l$. Then $\xi \in \operatorname{Aut}(S, \sigma)$ so $\operatorname{Aut}(S, \sigma)$ is a normal subgroup in all three cases.

COROLlaRY 4.5. Suppose $(M\{,\},, \mathcal{L}, b, \phi)$ is a basic LMTS with $\phi$ symmetric or symplectic. If $\Lambda_{w} \subseteq \Lambda_{r}, \operatorname{Aut}(S, \sigma) \cong \operatorname{ker} \bar{r} \times \operatorname{im} \bar{r}$.

Proof. This follows from Theorem 3.1.

Note 4.5. When studying the forms of a Type (III) LMTS $(M\{,\},, \mathcal{L}, b, \phi)$ in a subsequent paper, we will need to consider the groups $\operatorname{Aut}^{*}(\bar{S}, \sigma):=\{\eta \in$ $\operatorname{Aut}(\bar{S}, \sigma) \mid M_{i} \eta=M_{i}$ for $\left.i=1,2\right\}$ and $\operatorname{Aut}^{*}(S, \sigma):=\operatorname{Aut}(S, \sigma) \cap \operatorname{Aut}^{*}(\bar{S}, \sigma)$ and also the homomorphisms $\bar{r}^{*}: \operatorname{Aut}^{*}(\bar{S}, \sigma) \rightarrow \operatorname{Aut} \mathcal{L}$ and $r^{*}: \operatorname{Aut}^{*}(S, \sigma) \rightarrow$ Aut $\mathcal{L}$ 
given by restriction. The results obtained already give the following statements about these groups: $\operatorname{ker} r^{*} \cong k^{*}, \operatorname{ker} \bar{r}^{*} \cong k^{*} \times k^{*}, \operatorname{Inn} \operatorname{Aut} \mathcal{L} \subseteq \operatorname{im} r^{*}=\operatorname{im} \bar{r}^{*}$, and $\operatorname{Aut}^{*}(S, \sigma)$ is a normal subgroup of $\operatorname{Aut}^{*}(S, \sigma)$ with $\operatorname{Aut}^{*}(\bar{S}, \sigma) / \operatorname{Aut}^{*}(S, \sigma) \cong k^{*}$. If $\eta \in \operatorname{Aut}^{*}(\bar{S}, \sigma)$ such that $\eta$ stabilizes a Borel subalgebra $B$, then $\lambda_{i} \eta^{*}=\lambda_{i}$ for $i=1,2$. Either $\operatorname{Aut}^{*}(\bar{S}, \sigma)=\operatorname{Aut}(\bar{S}, \sigma)$ or $\operatorname{Aut}^{*}(\bar{S}, \sigma)$ is a normal subgroup of $\operatorname{Aut}(\bar{S}, \sigma)$ of index 2. If $a \neq \pm 1, \operatorname{Aut}^{*}(S, \sigma)=\operatorname{Aut}(S, \sigma)$.

\section{REFERENCES}

1. N. C. Hopkins, Decomposition of Lie module triple systems, Algebras, Groups and Geometries (to appear).

2. _ , On the derivation algebras of Lie module triple systems, J. Algebra (to appear).

3. 203-212.

4. N. Jacobson, Lie algebras, Dover, New York, 1962.

5. W. Lister, A structure theory of Lie triple systems, Trans. Amer. Math. Soc. 72 (1952), 217-242.

6. R. Steinberg, Lectures on Chevalley group, Yale Univ. Notes, New Haven, Conn., 1967.

Department of Mathematics, The Ohio State University, Columbus, Ohio, 43210-1174

Current address: Department of Mathematics and Computer Science, Indiana State University, Terre Haute, Indiana 47809 